\title{
Practical application of the European Field Guide in screening for diabetic retinopathy by using ophthalmoscopy and $35 \mathrm{~mm}$ retinal slides
}

\author{
R.L. Gibbins, D. R. Owens, J. C. Allen, L. Eastman \\ Diabetes Research Unit, University of Wales College of Medicine, Llandough Hospital, Penarth, Wales, UK
}

\begin{abstract}
Summary The Welsh Community Diabetic Retinopathy Study was designed to assess the effectiveness of the Field Guide Book for screening for diabetic retinopathy in Europe. A community-based sample (prevalence $2 \%$ ) of diabetic patients was recruited from four general practices. Standardised training and equipment were provided. All patients were invited to attend practice-based screening sessions on two occasions over 3 years (phases 1 and 2). After mydriasis, clinical ophthalmoscopy was performed by a study optometrist and general practitioners (GPs). $2 \times 45^{\circ}$ field $35 \mathrm{~mm}$ retinal slides were obtained according to EURODIAB protocol. Anonymised slides were assessed by GPs, diabetologists and the optometrist. All the findings were graded externally (reference standard). In phase 2 community optometrists also performed ophthalmoscopy and assessed photographs. For detecting sight threatening diabetic retinopathy using ophthalmoscopy, GPs
\end{abstract}

achieved a sensitivity of $65.7 \%$, specificity $93.8 \%$ and positive predictive value (PPV) $65.7 \%$. Community optometrists achieved a sensitivity of $82.2 \%$ with a PPV of $50.7 \%$; the study optometrist 79.2 and $55.9 \%$, respectively. The use of $35 \mathrm{~mm}$ slides improved sensitivity for the detection of sight threatening retinopathy to $87.3,91.1$ and $97.2 \%$ for GPs, community optometrists and the study optometrist, respectively. PPV fell to $51.2 \%$ for GPs, $40.6 \%$ for community optometrists, but increased to $58.8 \%$ for the study optometrist. Diabetologists achieved a sensitivity of $88.7 \%$ and a PPV of $65.6 \%$. It is concluded that the European field guide is an effective tool for screening for retinopathy in clinical practice. [Diabetologia (1998) 41: 59--64]

Keywords Diabetic retinopathy, screening, ophthalmoscopy, photography, general practitioner, optometrist.
A primary objective of the St. Vincent declaration [1] is to achieve a significant reduction of blindness due to diabetes mellitus. Screening for retinopathy before visual loss has occurred is essential in achieving this

Received: 6 May 1997 and in revised form: 8 August 1997

Corresponding author: Dr. R.L. Gibbins, Diabetes Research Unit, University of Wales College of Medicine, Academic Centre, Llandough Hospital, Penlan Road, Penarth, South Glamorgan CF64 2XX, UK

Abbreviations: CO, Community optometrist; DR, diabetic retinopathy; DRGC; Diabetic Retinopathy Grading Centre; GPs, general practitioners; PPV, positive predictive value; STDR, sight threatening diabetic retinopathy; WCDRS, Welsh Community Diabetic Retinopathy Study; NIDDM, non-insulin-dependent diabetes mellitus. objective, and, as a consequence, a workshop was held at the Hammersmith Hospital, London, UK in October 1990 to define protocols for screening. Participants included representatives of diabetes and ophthalmological societies from many European countries. A Field Guide Book for clinical use in screening for diabetic retinopathy (DR) [2] was produced as a result of this workshop. It was recognised that whereas ideally ophthalmologists should carry out this work, in some countries where there are insufficient numbers, adequately trained staff of other grades could perform screening. This necessitated the definition of lesions requiring urgent ophthalmological attention to preserve sight (sight threatening DR (STDR)). Screening using direct ophthalmoscopy or retinal photography were considered acceptable, 
provided mydriasis was used. With photography, a $45^{\circ}$ camera taking two retinal fields, one centred on the macula and one nasal to the disc were recommended, to produce $35 \mathrm{~mm}$ transparencies. Instant (Polaroid) prints were deemed unacceptable because of poor definition and fading over time.

The methodology for $35 \mathrm{~mm}$ retinal photography was subsequently validated against accepted international standards derived from the Wisconsin Epidemiological Study of Diabetic Retinopathy (WESDR) [3] as part of the EURODIAB IDDM complications study [4].Validation included both the use of two field $45^{\circ} 35 \mathrm{~mm}$ retinal slides with very minor variations in field definition and a clinical grading system for retinopathy compatible with the European protocols detailed in the Field Guide Book [2]. The use of two $45^{\circ}$ fields approximate to four $30^{\circ}$ fields of the standard seven used in WESDR. Two or four $30^{\circ}$ retinal fields have shown $80 \%$ and $91 \%$ agreement respectively in grading of eight levels of DR, and up to $95 \%$ for four levels of DR, compared with the standard seven fields [5].

The standards by which screening programmes should be assessed have not been defined in the European protocols. Computer modelling for insulindependent diabetes mellitus has suggested that a threshold sensitivity of $60 \%$ for any retinopathy would be most cost beneficial with little to be gained from an increase to $80 \%$ [6]. This analysis assumed that all subjects with known retinopathy were reviewed at 6 monthly intervals. A more recent study [7] based on United Kingdom models has indicated that screening interval may be a significant factor at sensitivities between 52 and $67 \%$. However, a symposium held in Exeter, UK in 1994 under the auspices of the British Diabetic Association suggested that, in clinical practice, screening techniques should achieve a sensitivity of at least $80 \%$ and a specificity of $95 \%$ with no more than a $5 \%$ technical failure rate [8]. Most groups involved in screening in the United Kingdom have been working to these minimum standards since then. Positive predictive value (PPV) -the 'true positive' percentage of those referred -- is a useful indicator of effective screening, and a minimum PPV of $55 \%$ would correlate with the proposed minimum sensitivity and specificity.

While there is agreement on the need to screen for DR and the standards to be achieved, debate continues as to the most appropriate methods. In the United Kingdom various practical programmes have been established, though the methodology and reference standards used have not been standardised. The use of mobile retinal cameras is widespread, and experience in their use has recently been summarised [8]. Data on the prevalence of retinopathy was not available in this study though $5.6 \%$ of those screened were referred for ophthalmological advice. The true prevalence of STDR is unknown but current evidence suggests that $40--45 \%$ of the diabetic population have some DR with 10--14\% having STDR $[9,10]$. If this is so, it is possible that mobile photography is missing a significant number of people with STDR. Similarly, studies employing direct ophthalmoscopy carried out by general practitioners (GPs) and optometrists in the United Kingdom [11--14] have found a prevalence of STDR of 4.9--7.1\% and in the Netherlands GPs using ophthalmoscopy achieved a sensitivity of only $52 \%$ [15], suggesting that this method may also be inadequate. None of these studies employed a retinopathy grading system based on European standards or otherwise validated.

The Welsh Community Diabetic Retinopathy Study (WCDRS) was designed to address some of these outstanding issues by being community based and using standardised equipment and training methods for all assessors of retinopathy. The study also employed examination techniques and a retinopathy grading system derived directly from the European Field Guide, allowing us to report on the practical application of the European protocols in a clinical setting.

\section{Subjects and methods}

Data from a pilot study [16] indicated that at least 600 subjects would be required to achieve confidence limits on the sensitivity of detection of any DR of $\pm 3 \%$ and STDR of $\pm 7 \%$. Four Welsh group general practices participated in the study, providing approximately 1000 subjects. Practices were required to have a known prevalence of diabetes of at least $2 \%$, a computerised diabetes register and facilities for on-site clinical screening sessions, though none had pre-existing screening programmes for diabetic retinopathy.

All registered patients with diabetes in the study practices were invited to attend screening sessions in their GP's premises on two occasions (phases 1 and 2) over a 3-year period. Mydriasis using $1 \%$ tropicamide was universally employed in these sessions unless subjects were known to have glaucoma or a history of anterior lens implantation (with a risk of lens dislocation), when examination was carried out with undilated pupils. When adequate pupil dilation was obtained, direct ophthalmoscopy was carried out by the participating GP, followed by direct ophthalmoscopy and retinal photography conducted by a specialist optometrist (S. W.), who, in addition to a general optometric qualification, had received special training in the identification and grading of diabetic retinopathy. EURODI$\mathrm{AB}$ protocols [4] were used to obtain two $45^{\circ}$ retinal fields per eye, one centred on the macula, the other nasal to the optic disc, in the form of $35 \mathrm{~mm}$ colour transparencies using Kodak Ektachrome Elite 100 film, mounted as slides. Within 2 weeks of the second clinic visit, participants were offered an appointment with a non-specialist community optometrist currently providing routine refraction and eye examination services and practicing in the same geographical area as their GP. Optometrists conducted direct ophthalmoscopy according to the same protocol as GPs.

Anonymised retinal slides were examined and graded for DR and STDR by six GPs from the participating practices and a specialist optometrist (S. W.) (phases 1 and 2), four consultant physicians with a specialist interest in diabetes (diabe- 


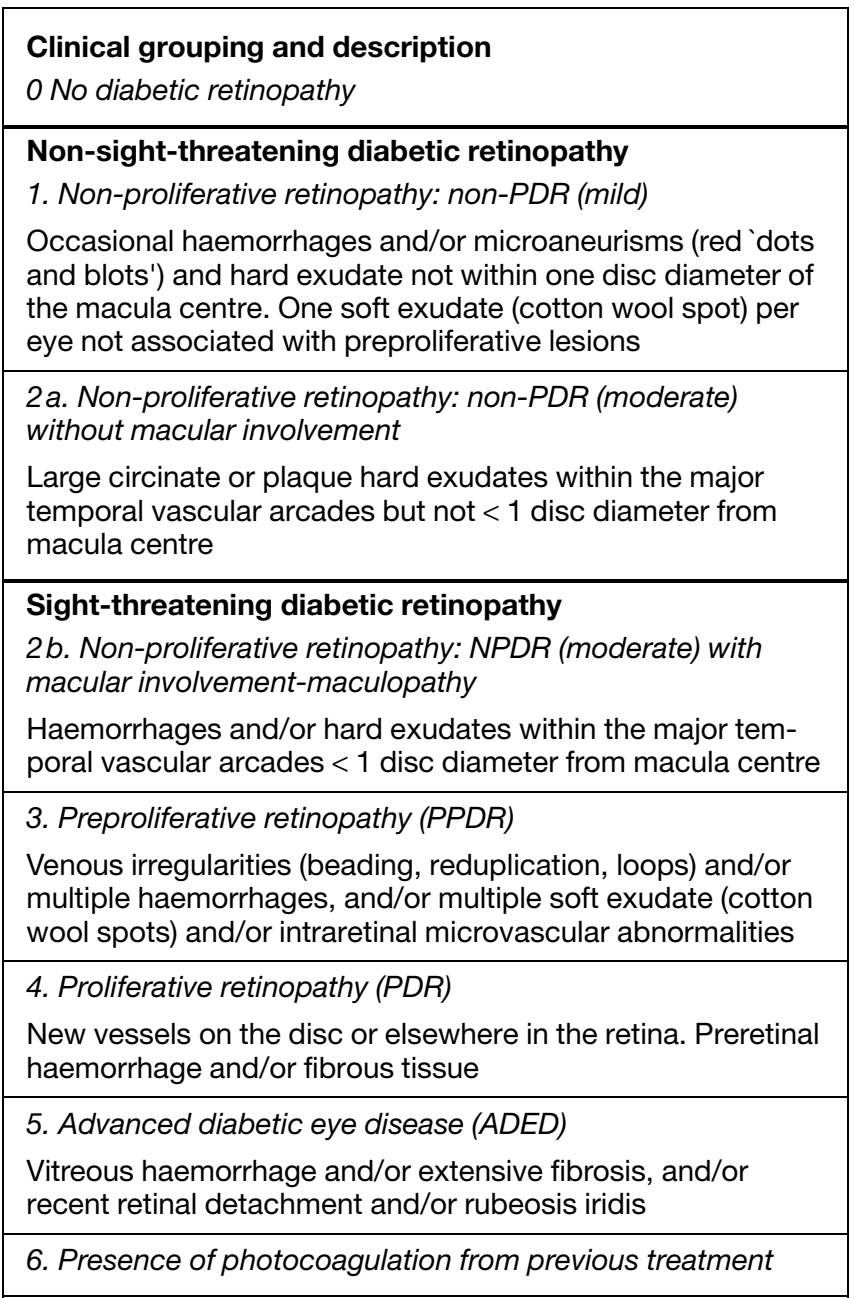

Fig. 1. Welsh community diabetic retinopathy study clinical groupings

tologists) recruited from district general hospitals in South Wales (phase 1) and the 11 community optometrists who also conducted clinical examinations (phase 2). GPs and community optometrists only assessed retinal slides of subjects on whom they had carried out direct ophthalmoscopy. The specialist optometrist assessed all retinal slides. The diabetologists each examined $25 \%$ of the pooled retinal slides.

All assessors were provided with standardised modular training. The core element of training -- the natural history of DR and identification and grading of DR according to study protocols -- was the same for all assessors. GPs and optometrists received additional training according to specific needs. For GPs this included correct use of the ophthalmoscope, and for optometrists a summary of the pathophysiology of diabetes. GPs were provided with good quality ophthalmoscopes (Welch Allyn, Skaneateles falls N. Y., USA), and all assessors used the same slide viewing boxes (Slidex, Tokyo, Japan) for grading retinal slides.

The WCDRS retinopathy grading system (Fig. 1) employed by all assessors for both direct ophthalmoscopy and examination of slides was derived directly from European protocols [17] summarised in the European Field Guide [2], and agreed in advance with the Diabetic Retinopathy Grading Centre (DRGC) at the Hammersmith Hospital, London. Quality assessment of slides employed EURODIAB criteria [4] with four quality levels as follows:
Level 1: Excellent -- full-field photograph, picture definition good, lesions easily discernible as present or not present;

Level 2: Good -- 3/4 field photograph, picture definition good, lesions are easily discernible as present or not present; Level 3: Fair -- $1 / 2$ field photograph, picture definition good, lesion discrimination uncertain due to light or other aberration e.g. eyelashes;

Level 4: Poor -- Less than $1 / 2$ field photograph, picture definition poor, lesion discrimination poor.

The DRGC used the retinal slides to provide an external reference standard against which the performance of all assessors was judged. Two trained independent graders graded all slides, with a third senior grader providing adjudication when differences of opinion emerged. Internal review of all images was used on $20 \%$. Slides were graded using EURODIAB methodology [4] with a WCDRS clinical grade derived from the worst eye per patient.

The study protocol was approved by local ethical committees covering the study practice areas. All patients were provided with an information sheet and gave their written informed consent before acceptance into the study. To comply with ethical standards all retinal slides were viewed by one of the clinical co-ordinators (D.R.O., R.L.G.) within 1 week of being developed. Where STDR had been missed during ophthalmoscopy, GPs were quickly notified and patients referred according to the study guidelines.

Statistical analysis. Data were analysed using the computer software package SPSS for Windows, Version 6.0. As a reference standard was being employed to evaluate a diagnostic test used as a screening procedure, sensitivity (percentage of positives correctly identified), specificity (percentage of negatives correctly identified) and positive predictive value (percentage of diagnosed positives correctly identified, PPV) were used as the appropriate statistics. The Confidence Intervals Analysis (CIA) computer software programme was used to calculate $95 \%$ confidence intervals.

Results are presented for the GPs and the specialist optometrist from phase 1 , the diabetologists from phase 1 , and the community optometrists from phase 2. GP and specialist optometrist results from phase 2 are not included in the analysis because they were not directly comparable with those of the diabetologists and community optometrists since the former had by then received additional training and experience.

\section{Results}

At the start of phase 1 of the study there were 959 identified diabetic patients out of a total study population of 47462 , giving a known prevalence of $2 \%$ for diabetes. On medical grounds 62 subjects were excluded from attending. Of the possible attendees 613 $(68 \%)$ attended clinic sessions and 605 were photographed. Of those who attended $343(55.9 \%)$ were male, and $502(81.9 \%)$ had non-insulin-dependent diabetes (NIDDM). When phase 2 commenced there were 996 known diabetic patients (prevalence $2.2 \%$ ), 47 were excluded from attending on medical grounds. 644 (68\% of possible attendees) attended and 640 were photographed, $365(56.7 \%)$ of those who attended were male, and $513(79.7 \%)$ had NIDDM. 
Pre-existing eye disease was not a reason for medical exclusion. No systematic differences in prevalence or attendance were observed between the participating practices. Of the possible attendees $81 \%$ were seen in either phase 1 or phase 2 , and $47 \%$ attended both phases.

The prevalence of any DR determined by the DRGC was $43.6 \%$ in phase 1 and $39.4 \%$ in phase 2 . For STDR the prevalence was $14.6 \%$ in phase 1 and $10.4 \%$ in phase 2 . Of those only attending phase 2 $55 \%$ had been diagnosed since the commencement of phase 1 . Only $12.2 \%$ of these had any DR and $3.3 \%$ STDR, and $16 \%$ of those diagnosed as having STDR in phase 1 did not attend phase 2, explaining the reduction in DR and STDR prevalence observed between phases.

In phase $1,78.0 \%$ of slides and in phase $2,78.4 \%$ of slides were assessed by the reference centre as being either excellent (level 1) or good (level 2) quality. Of the remainder, only $1.3 \%$ in phase 1 and $2.0 \%$ in phase 2 were assessed as quality level 4 , and therefore ungradable.

For the subsequent analyses of sensitivity, specificity and positive predictive value (PPV), subjects have only been included where slides of excellent or good quality (levels 1 and 2) were available for the macular fields of both eyes. The reference standard for all assessors was the subject's worst eye as graded by the DRGC.

Table 1 shows the performance of GPs, community optometrists and the specialist optometrist in identifying any DR using ophthalmoscopy or $35 \mathrm{~mm}$ retinal slides, and diabetologists performance using slides only. For GPs, community optometrists and the specialist optometrist for whom both methods were assessed, the use of $35 \mathrm{~mm}$ slides consistently improved sensitivity by between $14.3 \%$ (community optometrists) and $16.6 \%$ (GPs). PPV also improved for both the GPs and specialist optometrist, but fell slightly for the community optometrists. PPV was highest at $89.9 \%$ for the diabetologists, but at a cost of a relatively low sensitivity of $72.6 \%$. The specialist optometrist achieved the best overall performance in detecting any DR when using $35 \mathrm{~mm}$ slides, achieving a sensitivity of $86.1 \%$, a specificity of $88.9 \%$ and a PPV of $86.5 \%$.

Table 2 shows the ability of all observers to detect STDR using ophthalmoscopy or $35 \mathrm{~mm}$ retinal slides. Community optometrists achieved, and the specialist optometrist almost achieved, the $80 \%$ sensitivity threshold using ophthalmoscopy, but all observers improved their sensitivity considerably when $35 \mathrm{~mm}$ slides were employed, all exceeding the $80 \%$ threshold. The most marked improvement was seen for GPs whose sensitivity increased from 65.7 to $87.3 \%$, though their PPV fell from 65.7 to $51.2 \%$. Community optometrists recorded the lowest PPV using $35 \mathrm{~mm}$ slides at $40.6 \%$. Diabetologists' sensitivity using

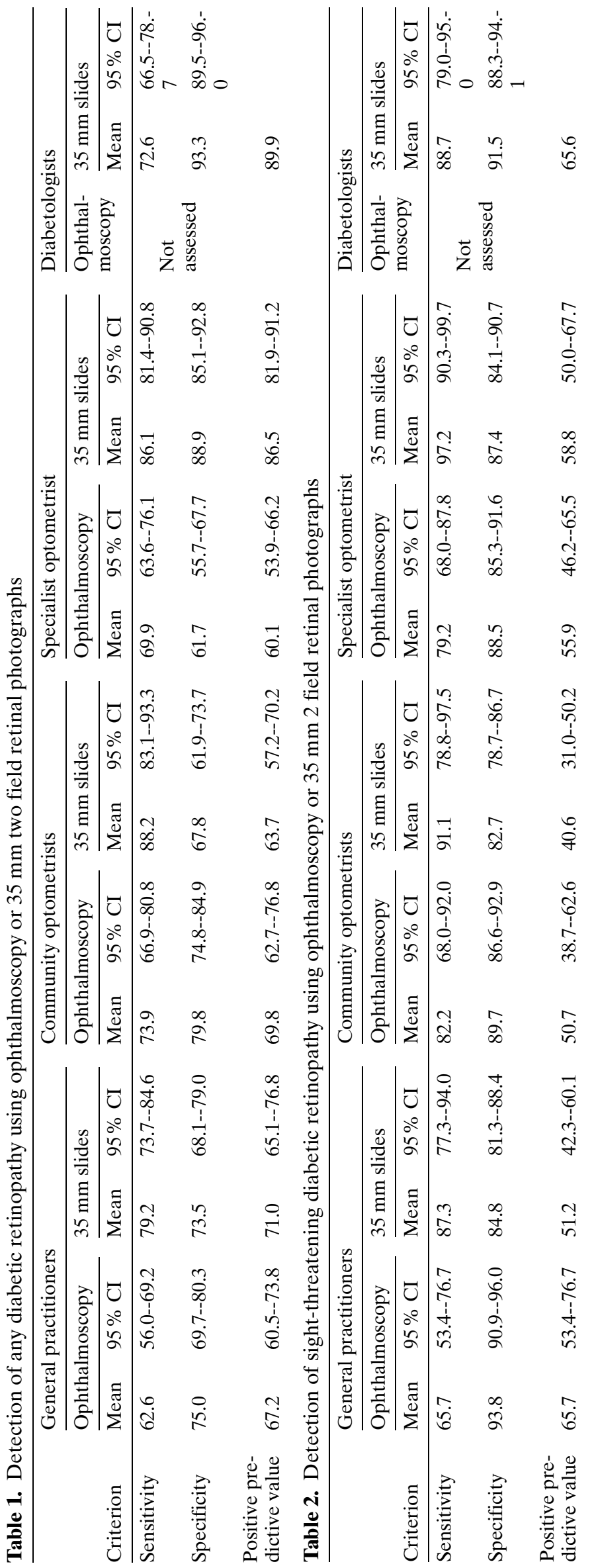


$35 \mathrm{~mm}$ slides $(88.7 \%)$ was similar to that of GPs, but their PPV was better at $65.6 \%$. The specialist optometrist again performed best using $35 \mathrm{~mm}$ slides, with a sensitivity of $97.2 \%$, specificity of $87.4 \%$ and PPV of $58.8 \%$

\section{Discussion}

In the United Kingdom, as for many other European countries, there are insufficient numbers of ophthalmologists to perform regular eye screening for all diabetic patients. There is some evidence [18, 19] that ophthalmologists screening for DR using direct ophthalmoscopy or slit lamp examination performed poorly (sensitivity $33 \%$ ) compared with internists using single $45^{\circ}$ slides (sensitivity $54 \%$ ) and retinal specialists using two stereo $45^{\circ}$ slides (sensitivity $81 \%$ ). Even in those countries where there are sufficient ophthalmologists, it is questionable whether screening for diabetic eye disease represents a cost efficient use of expensive specialist time if effective and more economical methods are available.

The Field Guide Book for Screening for Diabetic Retinopathy in Europe [2] is a consensus document reflecting a wide range of opinion among ophthalmologists and diabetologists. Our study was designed to provide evidence from clinical practice for the principles contained in the Field Guide Book. Established and validated European grading systems and protocols were employed using a community-based sample with a known high prevalence $(>1.8 \%$ ) of diabetes to compare the effectiveness of health professionals other than ophthalmologists in screening for DR.

In this study population the detection rates for any DR of 39.4--43.6\% and STDR of 10.4--14.6\% obtained by the reference standard are comparable with those obtained or derived from other reference populations $[3,10]$. This indicated that two $45^{\circ}$ photographic fields per eye in the form of $35 \mathrm{~mm}$ colour transparencies were, when assessed by accredited graders, an effective method of detecting all significant DR in the diabetic population.

Minor background retinopathy has little clinical significance in terms of visual loss, and therefore is of less importance than the detection of STDR in clinical practice. However, it was notable that the use of two field dilated $35 \mathrm{~mm}$ retinal slides improved the performance of GPs, COs and a specialist optometrist in detecting the presence of any DR, with sensitivities increasing by over $14 \%$ in all groups and the number of false positives falling for GPs and the specialist optometrist. The relatively low sensitivity achieved for any DR by diabetologists using $35 \mathrm{~mm}$ slides may result from high selectivity, reflected in the PPV approaching $90 \%$.
A similar pattern is seen when comparing the two methods for the detection of STDR, though there are more pronounced variations in PPV. In detecting sight threatening DR using ophthalmoscopy, GPs sensitivity $(65.7 \%)$ was poor compared with community optometrists $(82.2 \%)$ and the specialist optometrist $(79.2 \%)$, whereas GPs obtained the highest specificity $(93.8 \%)$ and PPV $(65.7 \%)$. This was a similar performance to that of an ophthalmologist 'in training' who achieved a sensitivity of $65 \%$ and specificity of $97 \%$ for STDR using ophthalmoscopy [7]. Data from the second phase of this study has indicated that, with increasing experience, GPs sensitivity for the detection of STDR using ophthalmoscopy improved to $81.3 \%$, with a PPV of $57.3 \%$, indicating an adequate performance.

The use of $35 \mathrm{~mm}$ slides improved sensitivity in the detection of STDR by $21.6 \%$ for GPs, $7.9 \%$ for community optometrists and $18 \%$ for the specialist optometrist. All three groups easily achieved the $80 \%$ threshold, but the low PPV of the community optometrists would result in a high level of overreferral in clinical practice. The marked improvement in performance by GPs resulted in a similar performance to that of the diabetologists, though the latter were more selective in identifying STDR as evidenced by their higher PPV. It was noteworthy that well-motivated community-based staff were able to achieve equivalent sensitivities to specialists in the detection of STDR, in contrast to previous evidence [20]. The best overall performance in the study was obtained by the specialist optometrist using $35 \mathrm{~mm}$ slides, correctly identifying $97 \%$ of cases of STDR with a reasonable PPV (58.8\%). All assessors found the clinical grading system (Fig. 1) easy to use and helpful in making management decisions relating to referral.

If the acceptable sensitivity level is reduced to $60 \%$ as suggested by cost benefit models [6], then all observers using all techniques achieve the minimum desirable standard for the detection of STDR. However, $35 \mathrm{~mm}$ retinal slides provide a much better sensitivity for all observers, and have other advantages, in providing a permanent record of retinal status available for comparison with later images, and allowing external quality control and assurance of screening programmes. They may also be digitised for easier storage and transmission to remote sites for assessment by specialists [21].

These results suggest that motivated and trained GPs and community optometrists, using $35 \mathrm{~mm}$ colour transparencies, could provide an effective screening service for diabetic retinopathy, though at a lower level of performance than that provided by a specialist assessor, represented in this study by a specially trained optometrist. Regular training and supervision to maintain quality would be a prerequisite of any community-based screening programme employing 
large numbers of GPs or community optometrists as retinal assessors, increasing service costs and complicating management.

Photographic-based screening programmes would allow community-based capture of retinal images, ensuring coverage of those subjects who do not regularly attend hospital clinics. Images could be assessed centrally by smaller numbers of dedicated staff, more easily permitting continuous quality assurance. The role of diabetologists may be as secondary screeners for intermediate grades of retinopathy, thereby attempting to reduce the number of false positives before onward referral to ophthalmologists.

We conclude that two field $35 \mathrm{~mm}$ retinal slides, taken and interpreted according to European Guidelines $[4,14]$, provide the method of choice for community-based screening for diabetic retinopathy, and have clear advantages over direct ophthalmoscopy by GPs and optometrists. The Field Guide Book for Screening for Diabetic Retinopathy in Europe [2], modified into the form of the WCDRS grading system (Fig.1), proved a practical method of retinal screening in United Kingdom clinical practice. Trained non-specialist medical and paramedical staff can be trained to use this technique to provide an adequate screening standard, but the best performance will be obtained by using dedicated photograph assessors, who do not need to be medically qualified.

Acknowledgements. The participating General Practitioners: Dr. A. E. Gray, Dr. N. Statham, Dr. M. Harrison, Dr. M. Heneghan, Dr. B. Bowden and Dr. S. Warren whose support, advice and co-operation, along with that of their practice staff, is gratefully acknowledged. Mr. S. Aldington (RPMS) provided retinal photography training for the study optometrist and aided the development of the grading system. The Media Resources Centre, University of Wales College of Medicine (UWCM) processed the film and the Department of Medical Computing and Statistics, UWCM managed the data.

The WCDRS is funded by the Department of Health through the Welsh Office. Additional support was received from The Nevill Hall Hospital Thrombosis and General Research Fund, and other staff of the Diabetes Research Unit, University of Wales College of Medicine. The project group acknowledges the valuable contribution of members of its Steering Committee.

\section{References}

1. Anonymous (1990) Diabetes care and research in Europe: The Saint Vincent declaration. Diabet Med 7: 360

2. Kohner EM, Porta M (eds) (1990) Screening for diabetic retinopathy in Europe: A field guide book. World Health Organisation/International Diabetes Federation

3. Klein R, Klein BEK, Moss SE, Davis MD, DeMets DL (1984) The Wisconsin Epidemiologic Study of Diabetic Retinopathy -- III: Prevalence and risk of diabetic retinopathy when age at diagnosis is 30 or more years. Arch Ophthalmol 102: 527--532

4. Aldington SJ, Kohner EM, Meuer S, Klein R, Sjolie AK (1995) Methodology for retinal photography and assessment of diabetic retinopathy: The EURODIAB IDDM complications study. Diabetologia 38: 437--444

5. Moss SE, Meuer SM, Klein R, Hubbard LD, Brothers RJ, Klein BE (1989) Are seven standard photographic fields necessary for classification of diabetic retinopathy? Invest Ophth Vis Sci 30(5):823--827

6. Javitt JC, Canner JK, Frank RG, Steinwachs DM, Sommer W (1990) Detecting and treating retinopathy with type I diabetes mellitus: A health policy model. Ophthalmology 97(4):483--495

7. Davies R, Sullivan P, Canning C (1996) Simulation of diabetic eye disease to compare screening policies. Brit J Ophthalmol 80: 1--6

8. Taylor R (1996) Practical community screening for diabetic retinopathy using the mobile retinal camera: report of a 12 centre study. Diabet Med 13: 946--952

9. Kohner EM, Porta M (1991) Protocols for screening and treatment of diabetic retinopathy in Europe. European Journal Ophthalmol 1(1):45--54

10. Harding SP, Broadbent DM, Neoh C, White MC, Vora J (1995) Sensitivity and specificity of photography and direct ophthalmoscopy in screening for sight threatening eye disease: the Liverpool diabetic eye study. BMJ 311: 1131--1135

11. Gilbert CE, Armstrong S, Burns-Cox C, Dean Hart JC (1982) Screening of diabetics by ophthalmic opticians. Trans Ophthal Soc UK 102: 249--252

12. Gatling W, Howie AJ, Hill RD (1995) An optical practice based diabetic eye screening programme. Diabet Med 12: 531--536

13. Finlay R, Griffiths J, Jackson G, Law D (1991) Can general practitioners screen their own patients for diabetic retinopathy. Health Trends 23(3):104--105

14. O'Hare JP, Hopper A, Madhaven C, et al. (1996) Adding retinal photography to screening for diabetic retinopathy: A prospective study in primary care. BMJ 312: 679--682

15. Reenders K, de Novel E, van den Hoogen H, van Weel C (1992) Screening for diabetic retinopathy by general practitioners. Scand J Primary Health Care 10: 306--309

16. Gibbins RL, Kinsella F, Young S, Saunders J, Owens DR (1994) Screening for diabetic retinopathy in general practice using $35 \mathrm{~mm}$ colour transparency fundal photographs. Practical Diabetes 11(5):203--206

17. Anonymous (1991) A protocol for screening for diabetic retinopathy in Europe. Retinopathy Working Party. Diabet Med 8(3):263--267

18. Pugh JA, Jackobson JM, Van Heuven WAJ, et al. (1993) Screening for diabetic retinopathy: The wide angle camera. Diabetes Care 16(6):1193--1195

19. Lairson DR, Pugh JA, Kapadia AS, Lorimor RJ, Jacobson J, Velez R (1992) Cost-effectiveness of alternative methods for diabetic retinopathy screening. Diabetes Care 15(10):1369--1377

20. Buxton MJ, Sculpher MJ, Ferguson BA, et al. (1991) Screening for treatable diabetic retinopathy: A comparison of different methods. Diabet Med 8: 371--377

21. Owens DR, Gibbins RL, George LD, Lusty J, Morton RA, Allen J (1997) Telemedicine in screening/monitoring of diabetic eye disease. Proceedings of current perspectives in healthcare computing. In: Richards E (ed) British Journal of Healthcare Computing, part 1 pp 166--171 